\title{
HIGH-SPEED AXIAL-FLUX PERMANENT MAGNET MICROMOTORS WITH ELECTROPLATED WINDINGS
}

\author{
Florian Herrault, Preston Galle, and Mark G. Allen \\ School of Electrical and Computer Engineering, \\ Georgia Institute of Technology, Atlanta, Georgia, USA
}

\begin{abstract}
This paper reports the design and microfabrication of electroplated multi-phase stator windings and their use in a high-speed permanent magnet micromotor. As the stator windings of an axialflux micromotor, both single-plated folded coils, and two-layer double-plated interleaved coils are demonstrated. These stators, combined with a $10 \mathrm{~mm}$-diameter permanent magnet rotor and an all-CMOS drive circuit, enabled speeds in excess of 50,000 RPM and 200,000 RPM, respectively, establishing a trade-off between performance and fabrication simplicity. At these speeds, the dissipated power in the microfabricated coils was approximately $1.81 \mathrm{~W}$ and $1.80 \mathrm{~W}$ for the folded and double-plated coils, respectively.
\end{abstract}

\section{INTRODUCTION}

Microscale electromagnetic energy conversion is a key component of many high-performance MEMS electromechanical actuators such as micro-relays, micro-pumps, and micromotors. The small size of a microscale electromechanical system puts limits on its throughput, in terms of electrical power as well as generalized application capacity. Operating electromechanical systems at faster speeds is a fitting way to increase throughput without losing size and integration advantages.

Miniaturized rotary machines are particularly apt for highspeed operation. The small mass and size of the rotors give very small imbalance moments, naturally allowing faster rotation within a given bearing load rating. Also many rotary applications, such as fluidic pumping, machining, and electricity generation, scale very well to high speeds.

However, fabrication simplicity must be a key requirement to further expand the development of MEMS-based electromechanical systems and potentially commercialize these devices. With regards to the research in permanent-magnet micro- motors, achieving high performance and high rotational speeds with complex motor designs and fabrication has often been favored over commercially-viable designs. More specifically, sequential plated layers with interleaved conductors, as used in [1-6] for micro-coil fabrication, have enabled the demonstration of MEMS motors/generators. Recently, microcoils on printed circuit boards (PCBs) have been used in micromotors [7], but rotational speeds and overall performance were inferior to the previously-mentioned devices.

In this paper, we present two micromotors that attain high rotational speeds. One micromotor uses a complex coil stator fabrication with interleaved windings, and demonstrates rotational speeds higher than that of previously-reported permanent magnet micromotors. The second micromotor, which presents the same coil parameters, is constructed with an eye towards mass-manufacturability, using a simpler approach based on folding coil phases one atop the other.

The folded-coil stator fabrication process is detailed, followed by the motor driver topology. Experimental characterization is presented and includes maximum rotational speed measurements, and power dissipated in the coils.

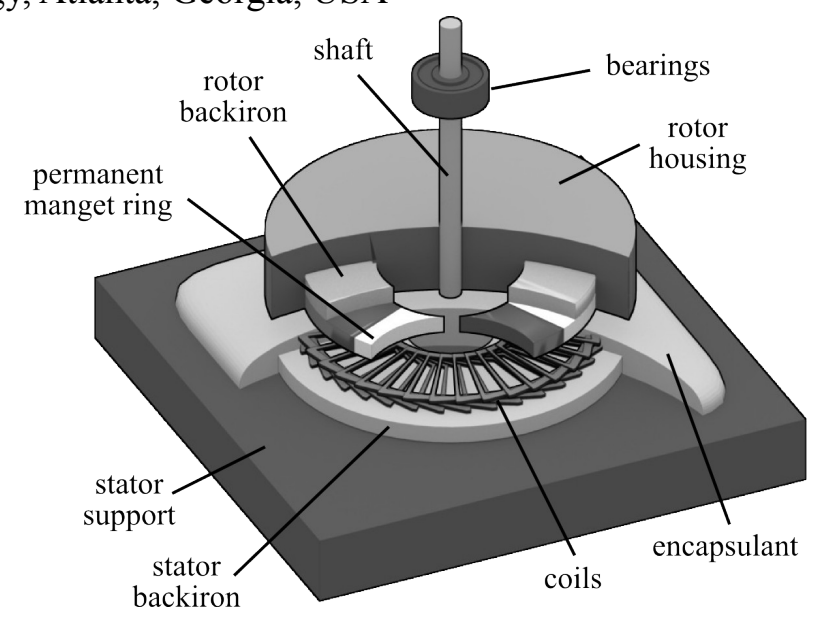

Figure 1: Cutaway view of generalized axial-flux motor

\section{Motor Design}

The motors presented in this research are largely identical to the generator presented in [1]. The axial-flux micromotors consist of an eight-pole permanent magnet rotor and a three-phase, three turns-per-phase stator with electroplated windings. A rendering of the device is shown in Figure 1. The $10 \mathrm{~mm}$-diameter rotor includes a $0.5 \mathrm{~mm}$ thick SmCo permanent magnet ring and a $0.5 \mathrm{~mm}$ thick $\mathrm{FeCoV}$ backiron encased in a titanium housing. A

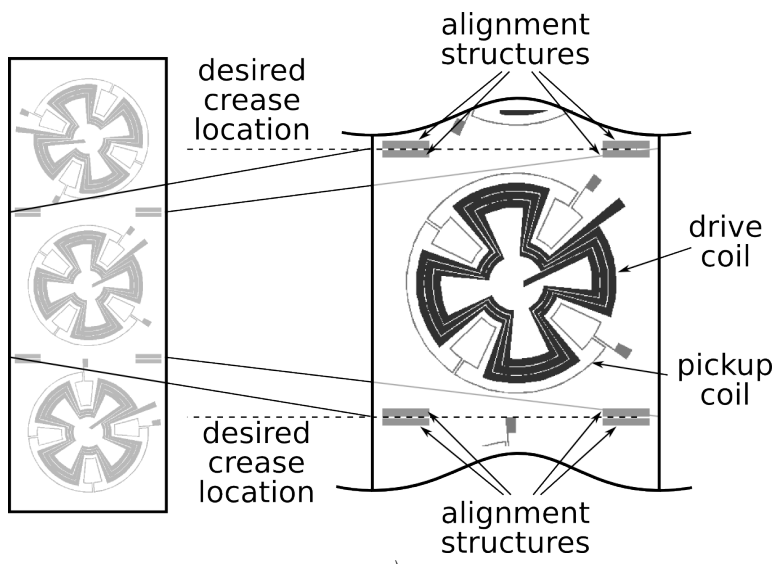

a)

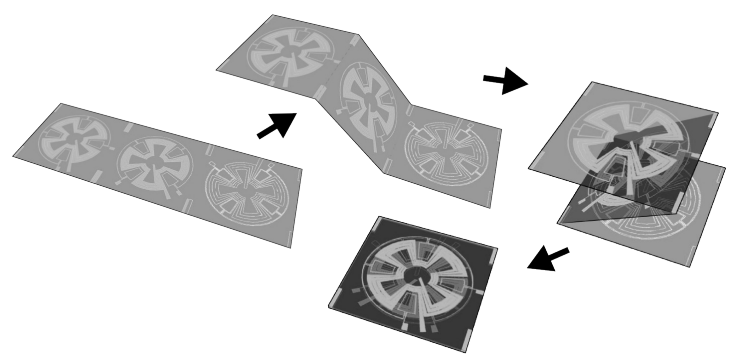

b)

Figure 2: Folded-coil a) stator design and b) folding process 
$1.6 \mathrm{~mm}$ diameter shaft connects the rotor to the turbine bearings for experimental characterization.

Two stator designs, denominated as folded-coil and doubleplated stators, were fabricated and tested, as reported in this paper. The fabrication of previously-reported windings, including the double-plated stator characterized in this work, requires multiple electroplating steps as well as patterning of intervening insulation layers, with careful attention to alignment and planarity as the layers accumulate [1-7]. While these multi-layer microfabrication techniques are now part of standard MEMS processes, they are still time-consuming and also very challenging to transfer to mass-production because of yield requirements. In contrast, the presented folding-based approach only requires a single electroplating step and blanket insulator depositions, while a folding step achieves excellent interlayer alignment. The three folded stator coils possess the same geometry as those in the double-plated stator: eight poles, three turns per phase, three phases, identical conductor widths, and inner and outer diameters of approximately $3 \mathrm{~mm}$ and $15 \mathrm{~mm}$, respectively.

\section{STATOR FABRICATION}

\section{Folded-coil stator}

The folded-coil stator was formed in three primary steps. First was the fabrication of a parylene sheet with embedded, single-layer electroplated copper structures. Second was the folding of this parylene sheet to form the multilayer stator. Finally, the folded structure, along with a $0.5 \mathrm{~mm}$ thick ferromagnetic backiron, were affixed into a stator housing with epoxy.

The plating mask for the folded coils is shown in Figure 2a,
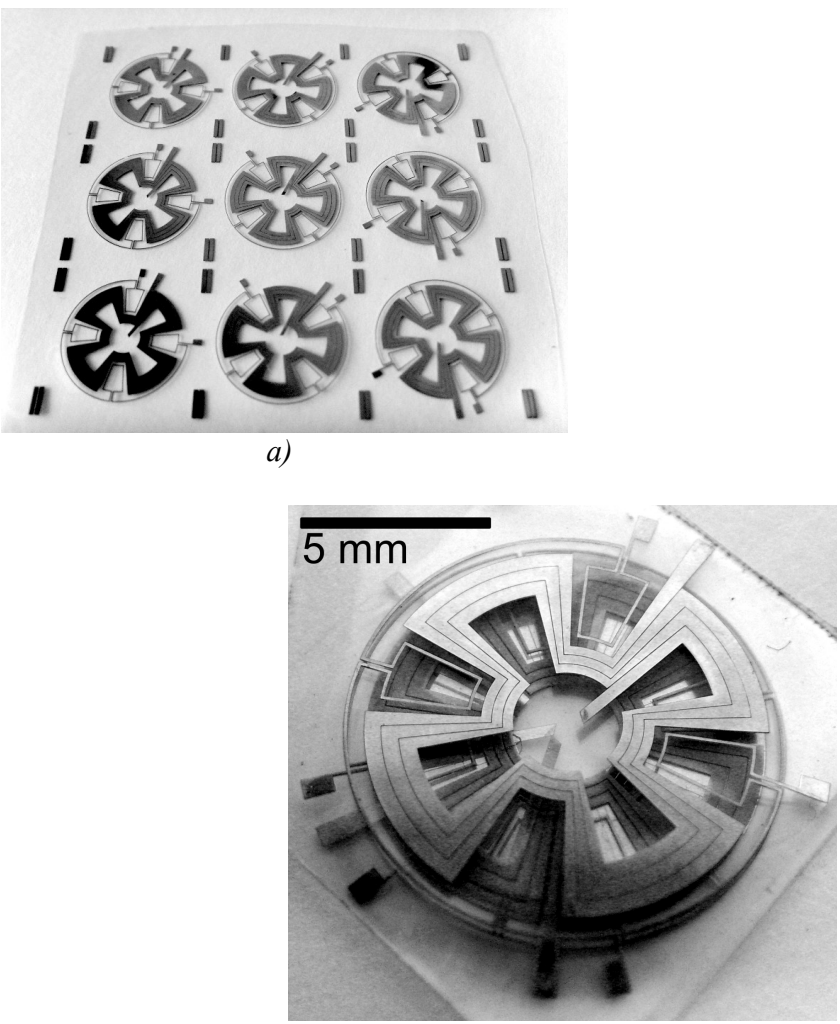

b)

Figure 3: Folded-coil fabrication results. a) shows an uncut, unfolded parylene sheet containing coils for 3 stators; b) shows a finished 3-layer folded coil and the folding process is illustrated in Figure 2b. Along each intended fold line there are four oblong rectangles. These are fold alignment structures and are electroplated along with the coils. They are essential to obtaining desired interlayer alignment, but after folding, they also provide substantial detent about the fold line, passively helping the stator maintain its fully folded state.

When folded, the three copper coils, which have similar geometries, are mechanically oriented at $30^{\circ}$ between one another. This is a design requirement in eight-pole machines to obtain an electrical angle of $120^{\circ}$ between the three phases. It should also be noted that pickup coils were designed and integrated within the coil stator topology, as pointed out in Figure 2a. These pickup coils will eventually provide position feedback for closed-loop control.

Conductors were fabricated by through-resist electrodeposition of copper onto an oxidized silicon substrate with a titaniumcopper-titanium seed layer. Futurrex NR-2 was used to construct the plating mold through which $80 \mu \mathrm{m}$ of copper was electrodeposited from an aqueous bath. After photoresist and seed layer removal, $6 \mu \mathrm{m}$ of parylene was deposited. The parylene-coated coils were then fully released by under-etching the oxide layer of the silicon substrate with an extended hydrofluoric acid etch (HF). Next, another $6 \mu \mathrm{m}$ parylene layer was deposited onto the newly exposed backside of the coils, rendering the structure fully passivated and ready for folding.

Figure $3 \mathrm{a}$ shows a finished parylene sheet containing three sets of coils, and Figure $3 \mathrm{~b}$ presents a finished three-layer winding. The coils exhibited an average resistance of $119 \mathrm{~m} \Omega$ and an average self-inductance of $0.56 \mu \mathrm{H}$.

\section{Double-plated stator}

The fabrication process for the double-plated stator was as in [1]. Two $80 \mu \mathrm{m}$ conductor layers, separated by a $40 \mu \mathrm{m}$ via layer, are electroplated atop a polyimide-coated high-permeability ferromagnetic (NiFeMo) substrate. The areal coincidence of interlayer vias with top conductor is exploited to combine these structures' depositions into a single electroplating step. In each electroplating step, copper is deposited onto a sputtered titanium-copper-titanium seed layer from an aqueous bath through a photoresist mold (Futurrex NR-9). The photosensitive epoxy SU-8 (Microchem) is used to fixate the plated copper after the plating mold and seed layer have been removed. The outcome, shown in Figure 4, is a stator

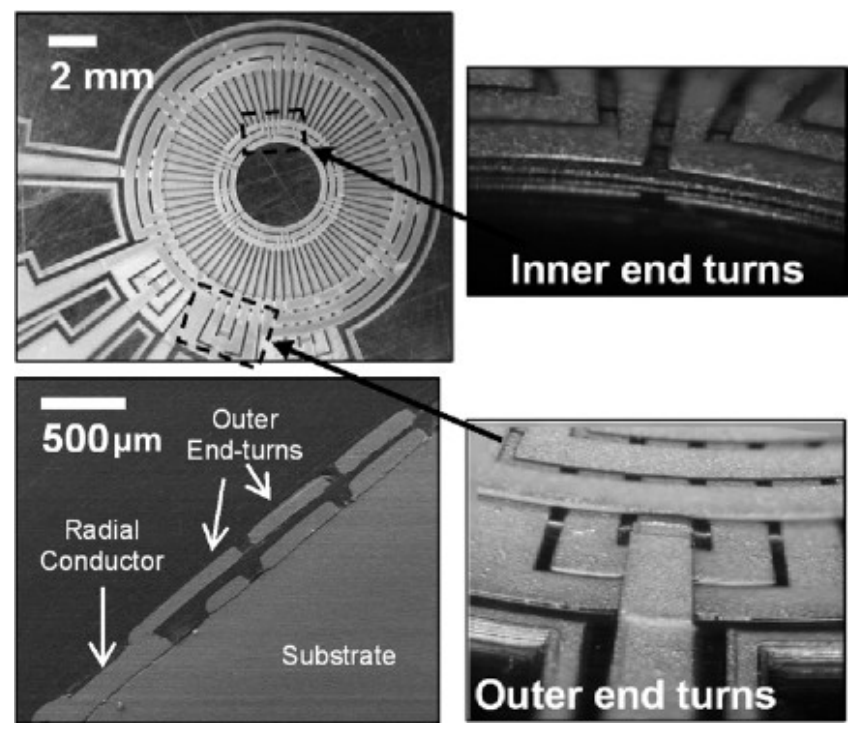

Figure 4: Double-plated stator (from [1]) 


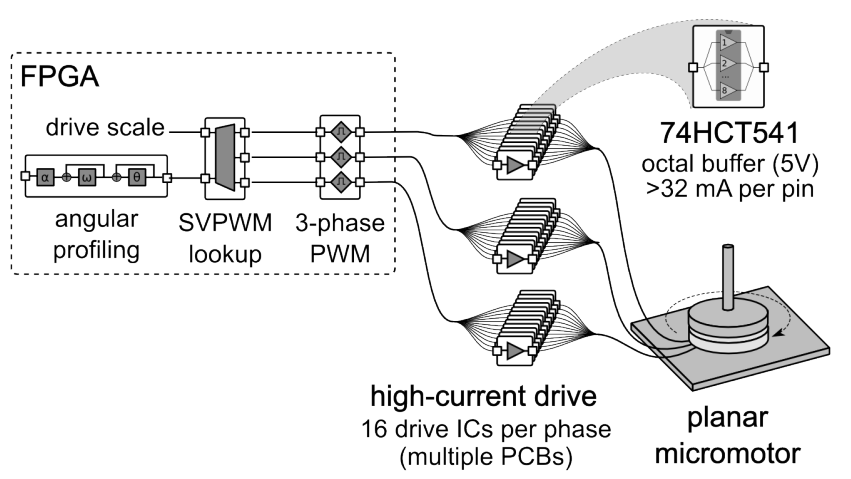

Figure 5: Motor driver system

structure consisting entirely of SU-8 and thick copper layers, with per-phase resistance of approximately $100 \mathrm{~m} \Omega$ and per-phase inductance of approximately $140 \mathrm{nH}$.

\section{MOTOR DRIVER DESIGN}

The motors were driven by the custom, all-CMOS system illustrated in Figure 5. Permanent magnet synchronous machines, such as the presented motors, are typically operated in a closedloop mode, relying on position feedback to drive electrical commutation. However, for this preliminary work, an open-loop control scheme was sufficient. The expected dominant mechanical loads, such as bearing friction and fluidic drag, present near-constant torque, and thus a minimal risk of sharp perturbations that would require dynamic correction. As well, the output drive amplitude could simply be temporarily increased, manually, during acceleration to ensure maximal immunity to perturbations.

Octal buffer ICs (Philips SN74HCT541) were chosen as output drivers. These fit the motor's need for very high drive frequencies, high drive currents and low drive voltages, but their built-in output current limiting also protects the microfabricated stators from overheating hazards. This implementation choice also clearly establishes the feasibility of driving the motor with conventional CMOS-based ASICs or full-custom ICs.

An open-loop space vector pulse width modulation (SVPWM) inverter was selected to generate the control signals for the output drivers. While allowing straightforward implementation in hardware, SVPWM provides compatibility with vector-based control, if closed-loop control is desired, and also minimizes the number of output switches. The controller was implemented in an Altera Cyclone III FPGA installed on a general-purpose development board (Terasic DE0). The output switching frequency and the commutation update rate was $1 \mathrm{MHz}$, nominally allowing rotation at well over one million RPM. The controller's governing parameters were exposed over an asynchronous serial port interface. A terminal communication program running on a PC served as the motor controller's user interface. A constant-acceleration angular profiler, with user-set parameters for speed and acceleration, generated the SVPWM's command angle. The SVPWM drive magnitude, or maximum duty cycle setting, was presented as a single user-set parameter.

To expedite characterization, an RL filter, not shown in Figure 5 , consisting of a $100 \mathrm{~m} \Omega$ resistor and a $5 \mu \mathrm{H}$ inductor, was placed in series with each motor phase. Phase currents were measured across the resistors. The inductors suppressed SVPWM switching currents, which were responsible for critical levels of both parasitic power dissipation in the stator and electrical noise in the current measurements.

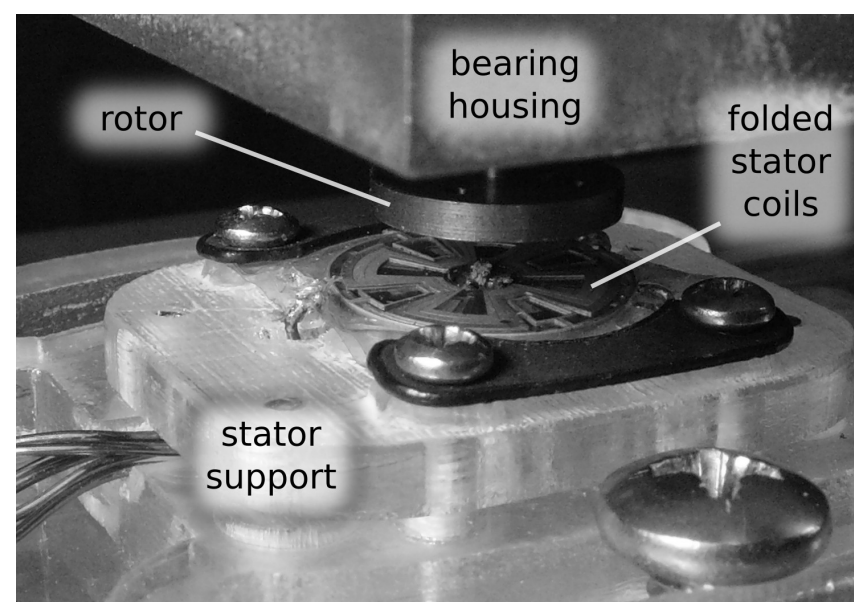

Figure 6: Photograph of micromotor with folded-coil stator

\section{CHARACTERIZATION}

Folded-coil stator

The folded-coil stator was first tested in generator mode, with compressed air driving a turbine attached to the rotor shaft. The stator housing was clamped onto $\mathrm{x}-\mathrm{y}-\mathrm{z}$ micro-positioners to ensure proper alignment between the stator and the rotor. A photograph of the assembled micromotor with folded coils is shown in Figure 6. The output waveforms for the three phases are shown in Figure 7. They exhibit excellent symmetry in both angle and magnitude, confirming proper coil alignment. The $10 \%$ discrepancy in magnitude is inherent to the design of the folded-coil stator. Because each phase is folded onto the others, the distance between each phase's coil and the stator ferromagnetic back iron varies. This introduces significant geometrical differences between the phases' magnetic circuits, naturally resulting in a variation in amplitude of their open-circuit voltages.

The stator was then characterized in motor mode. Maximum attainable speed was observed for a number of drive currents. During motor start-up and acceleration, the parameters for angular acceleration and drive magnitude were varied as needed. But at near-terminal speeds, the drive magnitude is held constant and the angular acceleration is set to very low value, under $500 \mathrm{RPM} / \mathrm{s}$. A reflective photoemitter-photodetector pair generated the tachometer signal used to measure the motor's speed.

These results are shown in Figure 8. The relationship clearly exhibits two plateaus. Below approximately $2 \mathrm{~A}$ per phase, the

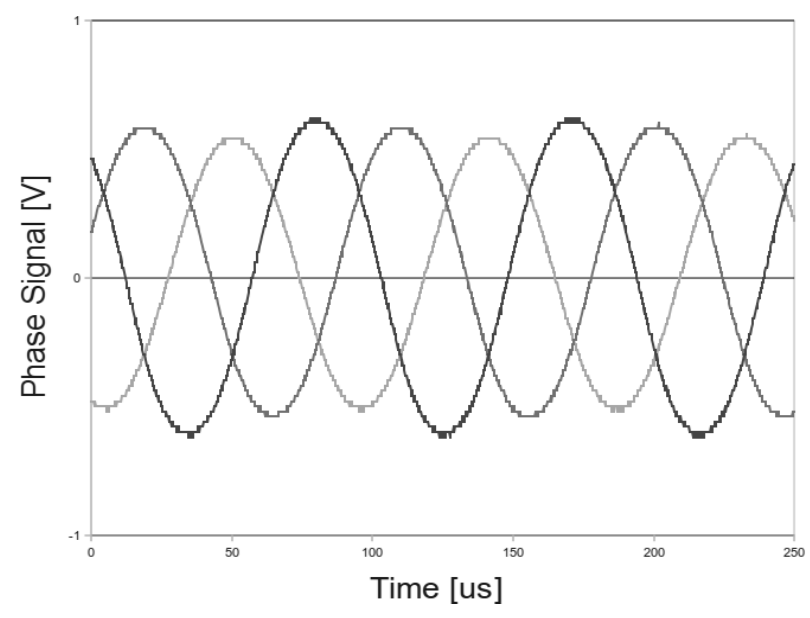

Figure 7: Folded-coil stator generator-mode waveforms 


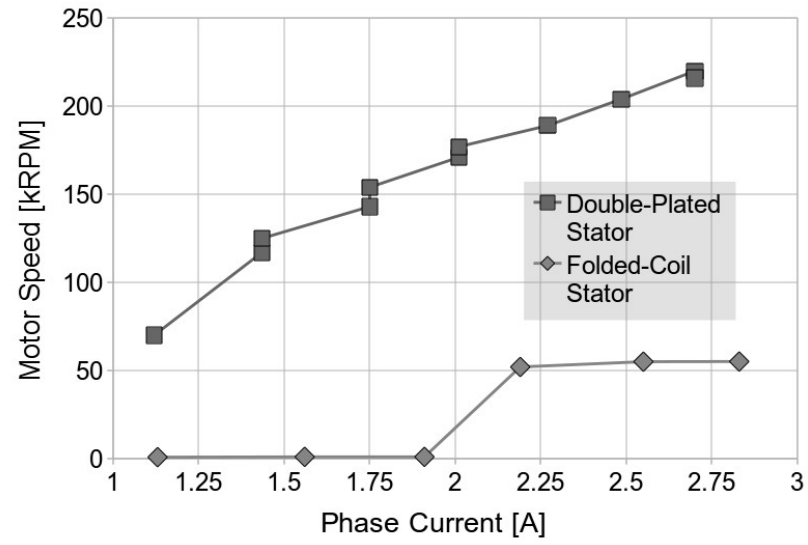

Figure 8: Maximum micromotor speeds for both stator types, for various drive currents.

motor rotated under 3000 RPM. At higher currents, the motor speed remains at approximately 50,000 RPM, reaching 52,000 RPM at a drive current of $2.83 \mathrm{~A}_{\mathrm{RMS}}$.

Although the nonlinearity in the speed-current graph was not investigated in detail, a likely cause is the high-speed bearings used. They are optimized for operation above 100,000 RPM, and exhibit several resonances at lower speeds.

\section{Double-plated stator}

The double-plated stator, having been characterized in generator mode in [1], was operated in motor mode. The experimental setup and procedure was identical to that used for the folded-coil stator. The results are also shown in Figure 8 for comparison. A speed of $204,000 \mathrm{RPM}$ at $2.49 \mathrm{~A}_{\mathrm{RMS}}$ per phase was attained. Under these operating conditions, the power dissipation in the coils was $1.86 \mathrm{~W}$.

The double-plated stator's performance was also measured as a function of stator-rotor gap. As exhibited in Figure 9, the micromotor operated best at a $400 \mu \mathrm{m}$ air gap. For smaller gaps, the magnetic pull-in force between the stator back iron and the magnetic rotor increased the axial load on the bearings, leading to higher bearing torque; larger gaps resulted in lowered magnetic interaction, producing less drive torque. Both phenomena result in lower maximum rotational speeds

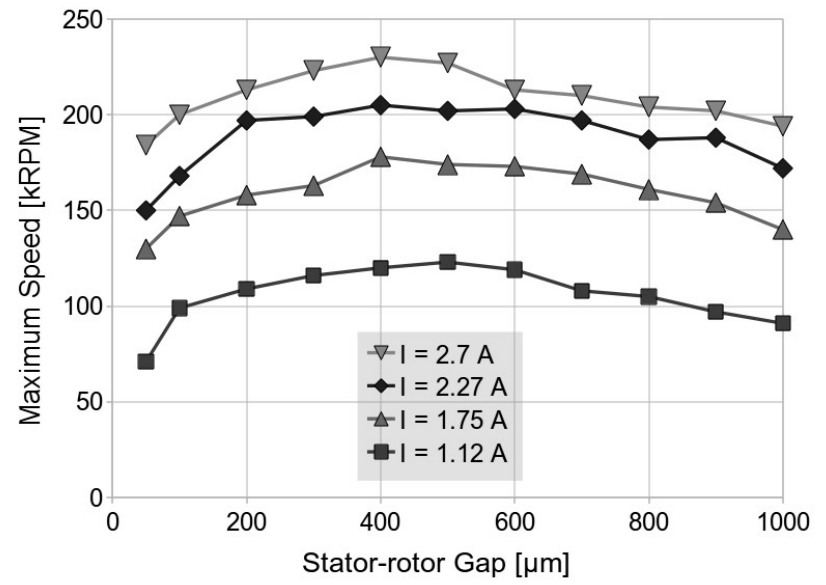

Figure 9: Influence of stator-rotor gap on performance of micromotor with double-plated stator
Heat accumulation imposed a major constraint on the achievable drive currents. The double-plated stator exhibited far better power dissipation capability than the folded-coil stator. This can be attributed to the double-plated stator's tighter vertical construction and thinner conductor to substrate insulation thickness, providing a lower thermal resistance for heat dissipation.

\section{CONCLUSIONS}

High-speed micromotors driven by an all-CMOS driver were presented in this paper, with an emphasis on two stator designs. Both of them had similar coil parameters (numbers of turns, poles, and phases), but one required an elaborate fabrication sequence using multiple electroplating steps, while the other one singled itself out by one electrodeposition and a folding technique. The two devices demonstrated high rotational speeds, indicating that folded micro-coils are suitable for efficient magnetic actuation at MEMS scales.

\section{ACKNOWLEDGEMENTS}

This project was supported by the DARPA MACE program and Raytheon Space \& Airborne Systems.

\section{REFERENCES}

[1] D. P. Arnold, F. Herrault, I. Zana, P. Galle, J.-W. Park, S. Das, J. H. Lang, and M. G. Allen, "Design Optimization of an 8 W, Microscale, Axial-flux, Permanent-magnet Generator," Journal of Micromechanics and Microengineering, 16, 9 (2006), pp. S290-6.

[2] F. Herrault, B. C. Yen, C.-H. Ji, Z. S. Spakovszky, J. H. Lang, and M. G. Allen, "Fabrication and Performance of Siliconembedded Permanent-magnet Microgenerators," Journal of Microelectromechanical Systems, 19, 1 (2010), pp. 4-13.

[3] C. H. Ahn, Y. J. Kim, and M. G. Allen, "A Planar Variable Reluctance Magnetic Micromotor with Fully Integrated Stator and Coils," Journal of Microelectromechanical. Systems, 2, 4 (1993), pp. 165-173.

[4] A. Waldschik and S. Büttgenbach., "Fabrication of Internal Driven Micro Centrifugal Force Pump Based on Synchronous Micro Motors with Polymer Magnet Rotors," 2009 Symposium on Design, Test, Integration \& Packaging of MEMS/MOEMS (2009 DTIP), Rome, Italy, 4/1-3/09, IEEE, Piscataway (2009), 4 pp.

[5] S. Merzaghi, C. Koechli, and Y. Perriard, "Development of a Hybrid MEMS BLDC Micromotor," Proceedings of the 2009 IEEE Energy Conversion Congress and Exposition (ECCE 2009), San Jose, CA, 9/20-24/09, IEEE, Piscataway (2009), pp. 3595-3601.

[6] N. Achotte, P.-A. Giles, O. Cugat, J. Delamare, P. Gaud, and C. Dieppedale, "Planar Brushless Magnetic Micromotors," Journal of Microelectromechanical Systems, 15, 4 (2006), pp. 1001-1014.

[7] D. Gambetta and A. Ahfock, "Designing Printed Circuit Stators for Brushless Permanent Magnet Motors," IET Electric Power Applications, 3, 5, (2009), pp. 482-90.

\section{CONTACT}

Corresponding author: Florian Herrault - 791 Atlantic Drive, Atlanta GA 30332 - (404-385-0273, fh59@mail.gatech.edu) 